\title{
The Dynamic Properties of a Nonlinear Economic Model with Extreme Financial Frictions
}

\author{
Huan Wang $\mathbb{D}$ and WenYi Huang \\ The School of Economic Mathematics, Southwestern University of Finance and Economics, WenJiang, Chengdu 611130, China \\ Correspondence should be addressed to Huan Wang; harrywang1213@gmail.com
}

Received 28 April 2018; Accepted 7 June 2018; Published 5 July 2018

Academic Editor: Xue-Jun Xie

Copyright (C) 2018 Huan Wang and WenYi Huang. This is an open access article distributed under the Creative Commons Attribution License, which permits unrestricted use, distribution, and reproduction in any medium, provided the original work is properly cited.

\begin{abstract}
A generalized economic model with two kinds of agents (farmers and landlords) is investigated. Farmers produce grains by renting lands from landlords. The land which is not rented is cultivated by less productive landlords. The economy is assumed to be with extreme frictions so that there are no markets for agents to trade grains. The rental rate is determined by the equilibrium of the supply and demand. We consider the situation that farmers are likely to take more risk when the rental rate is low and have more risk aversion if the rate goes high. The psychological anticipation is taken into account in the setting of our model. Using the optimal control theory, the dynamic properties of the rental rate and its influence to the endogenous volatility are analyzed. Besides, we clarify that the rental market has an instinctive ability to dominate the anticipation of agents.
\end{abstract}

\section{Introduction}

Since the Great Depression in 1933, many famous economists, such as Fisher and Keynes, have devoted themselves to the research of the failure of financial markets. Kindleberger [1] clarifies that financial crises are common in history. It seems that the financial frictions for business cycles play an important role in the crisis. Therefore, the desire to give more insight into the financial instability prompts scholars to set up new generalized continuous time equilibrium models. Earlier, Bernanke and Gerter [2] together with Kiyotaki and Moore [3] elaborate several important ways of how financial frictions influence the macroeconomy. One of their main conclusions is that small temporary shocks can have a great impact on the economy.

After the works of [3], He and Krishnamurthy [4, 5] build a kind of typical equilibrium models which allow us to give a research in the full-scale macroeconomic dynamics. Meanwhile, Brunnermeier and Sannikov [6] study the macroeconomic models and get further conclusions. Specifically, Brunnermeier and Sannikov show that the behaviour of the economy with aggregate shocks and financial frictions can be trapped in a less productive level for a long time. In Brunnermeier and Sannikov's model, the endogenous stochastic risks are set to discuss the behaviour of the economy. The feedback control techniques of stochastic nonlinear systems are found in [7-9].

Although Brunnermeier and Sannikov's work inspires us to study the behaviour of the macroeconomy in a new different way, there are also some drawbacks. Brunnermeier and Sannikov's model can only be solved in a numerical way and some of their predictions go somewhat against empirical evidence. To make up for these drawbacks, Klimenko et al. [10] have developed a dynamic macroeconomic model in a simple setting to analyze the significance of the endogenous risk. With the setting, they get dynamic equations and work out a quasi-closed form solution. They explicitly track down the roots of some important features like poverty trap, which is explained as the paradox of volatility and persistence of exogenous shocks. The implication of endogenous risk for welfare analysis is illustrated [10], while, in [10], they suppose an easy case in which the rental rate has a linear effect on its process. The assumption does not seem to be very practical. As will be illustrated carefully, we take the psychological anticipations of farmers into account and consider a nonlinear effect case.

Following the works in [10], we adopt two kinds of agents: risk-neutral landlords and risk-averse farmers. Landlords, 
which are less productive, own the land. Farmers cultivate grains by renting lands from landlords. In the economy, the financial friction is so high that there is no market for the agents to trade. Farmers have to keep the rest of the grains as their precautionary savings. To get rid of defaulting from their rent contracts, farmers have to adapt their scale of activity (the area of lands renting from landlords) to the level of the rental rate. The equilibrium of demand and supply in the rental market determines the process of the rental rate.

One of the remarkable settings in [10] is that the authors suppose there exists a rental market in which the rental rate follows a Markov stationary process, which takes the form similar to Geometric Brownian Motion (GBM). With the equilibrium conditions, Klimenko et al. [10] obtain the closed form solutions and consequently keep on with further analysis. The reason why they choose the form like GBM is that the rental rate is larger than 0 in the real world. Inspired by the works in [10], we take psychological anticipation of farmers into account. If the rental rate is low, farmers would choose to take more risk. On the contrary, farmers would be more risk averse if the rental rate is high. For this sake, the rental rate has an effect on the rental market and then influences its own process. The volatility fluctuates by the rental rate. We consider a rental rate process which differs from that in Klimenko et al. [10]. The closed form solution of the model is obtained. We show that the rental rate market has the ability to adapt itself even if we do not consider the psychological anticipation talked about above.

The rest of the paper is organized as follows. Section 2 gives a brief description of the model. Section 3 describes the equilibrium. Section 4 gives the ways to solve the equilibrium. Section 5 discusses its main properties. Section 6 studies the long-run average behaviour and analyzes the welfare loss problem. The conclusions are given in Section 7.

\section{Theoretical Framework}

2.1. Basic Statement of the Economy. In this section, we give the basic setting of the economy which is similar to that in [10]. There are only two types of agents in the economy: farmers and landlords. The landlords own the land, while the farmers can only rent it from the landlords. The rental rate $q_{t}$ is endogenously determined in the equilibrium. For convenience, the area of land in the economy is fixed and normalized to 1 . We assume that farmers are risk averse and landlords are risk-neutral. Both of the agents have the same discount rate $\rho$.

In the economy, the land which is cultivated by farmers yields more crop than that of landlords. More precisely, the flow of output yielding from one unit of land that is cultivated by a representative farmer is described by

$$
d y_{t}=a d t+\sigma_{0} d B_{t},
$$

where $a$ is the average growth rate of farmers and $\sigma_{0}$ stands for the aggregate shocks (like climate conditions) exposed in the economy. $B_{t}$ is a standard Brownian Motion defined on the probability space $(\Omega, \mathbb{F}, \mathrm{P})$ with the filtration $\mathbb{F}=\left\{\mathscr{F}_{t}, t \geq 0\right\}$.

It is hard to find a representative landlord. For this, we introduce a random variable $\widetilde{\alpha}$ which is continuously distributed over $[0, a]$. One unit of land cultivated by an $\tilde{\alpha}$ type of landlord yields the flow of output

$$
d \widetilde{y}_{t}=\widetilde{\alpha} d t+\sigma_{0} d B_{t} .
$$

In the economy, we assume there exists an extreme financial friction, which means that it is impossible for farmers and landlords to engage in any financial activities. The income of farmers comes from their producing activities and they have no other assets or resources. For some landlords, their productivity is so low that they might be likely to rent the land to farmers and gain rental rate $q_{t}$ from the farmers. For other landlords, they will cultivate by themselves. For farmers, they rent lands from landlords with rental rate if it is not so high. Because of the aggregate volatility, the productivity of the two agents varies. Hence, the equilibrium rental rent is fluctuated. In this paper, our goal is to find a Markov stationary equilibrium, where the rental rate $q_{t}$ satisfies the following Markov stochastic process:

$$
\frac{d q_{t}}{q_{t}}=\mu\left(q_{t}\right) d_{t}+\sigma\left(q_{t}\right) q_{t}^{\beta-1} d B_{t}, \quad 1 \leq \beta<2,
$$

where the drift $\mu\left(q_{t}\right)$ and diffusion $\sigma\left(q_{t}\right)$ are determined by the equilibrium conditions. The parameter $\beta$ is a constant which takes values between 1 and 2 .

As can be seen, the process in (3) is reduced to Klimenko et al. [10] if the parameter $\beta=1$. In Klimenko et al.'s model, the rental rate $q_{t}$ has a linear influence on the volatility $\sigma(q)$. Here we suppose that the rental rate process takes a form of the CEV model. The volatility is magnified when the rate is low. On the contrary, the volatility is reduced if the rate goes high. As will be clarified, the economy seems to have the instinctive ability to adapt itself whether we take the psychological anticipations into consideration or not.

\subsection{Decisions of Agents}

Farmers. At time $t$, each farmer chooses his scale of production $k_{t}$ and decides how much crop should be consumed, which is denoted by $c_{t}$. Hence, the saving of a representative farmer at time $t$, which is denoted by $s_{t}$, is driven by

$$
d s_{t}=k_{t}\left(d y_{t}-q_{t} d t\right)-c_{t} d t .
$$

In fact, both $k_{t}$ and $c_{t}$ depend on the scale of production and the rental rate.

All farmers in the economy are homogeneous and their utility function is logarithmic. Each farmer chooses his optimal consumption $c_{t}$ and scale of production $k_{t}$ to maximize his all-life expected discounted value of consumption

$$
V(s, q)=\max _{c_{t}, k_{t} \geq 0} E\left[\int_{0}^{+\infty} e^{-\rho t} \ln \left(c_{t}\right) d t \mid s_{0}=s, q_{0}=q\right],
$$

where $s$ is the farmer's initial stock of saving and $q$ is the initial rental rate.

Landlords. At each time $t$, every landlord decides whether to rent out his land or not according to the rental rate. If the 
rental rate is more than his own productivity, i.e., $q_{t} \geq \widehat{\alpha}$, he will rent the land out; otherwise he will cultivate by himself.

We assume that all landlords in the economy have infinite elasticity of intertemporal substitution. Each landlord will decide whether to rent the land out or not and consume all his savings immediately at each time $t$. Once given a rental rate $q_{t}$, the whole fraction of land leased by landlords is a continuous and increasing function of the rental rate, which is denoted by $K_{S}\left(q_{t}\right)$.

\section{Equilibrium}

In this section, we are supposed to find a way to solve the optimal control problem. Our basic idea is to find the solution of the HJB equation by dynamic programming principle.

Lemma 1. If $V\left(s_{t}, q_{t}\right)$ is the maximum expected discounted value of consumption on $(t,+\infty)$, then the following equation

$$
\begin{array}{r}
V(s, q)=\max _{c_{t}, k_{t} \geq 0} E\left[\int_{0}^{h} e^{-\rho t} \ln \left(c_{t}\right) d t\right. \\
\left.+e^{-\rho h} V\left(s_{h}, q_{h}\right) \mid s_{0}=s, q_{0}=q\right]
\end{array}
$$

holds.

Proof. On the temporal interval of $(0, h)$, we choose a control process of $c_{t}$ and $k_{t}$ evolves from $\left(0, c_{0}, k_{0}\right)$ to $\left(h, c_{h}, k_{h}\right)$.

(i) For any $h>0$, applying the tower property straightforward, we have

$$
\begin{aligned}
E & {\left[\int_{0}^{+\infty} e^{-\rho t} \ln \left(c_{t}\right) d t \mid \mathscr{F}_{0}\right] } \\
& =E\left\{E\left[\int_{0}^{+\infty} e^{-\rho t} \ln \left(c_{t}\right) d t \mid \mathscr{F}_{h}\right] \mid \mathscr{F}_{0}\right\} \\
& =E\left\{E \left[\int_{0}^{h} e^{-\rho t} \ln \left(c_{t}\right) d t\right.\right. \\
& \left.\left.+e^{-\rho h} \int_{h}^{+\infty} e^{-\rho(t-h)} \ln \left(c_{t}\right) d t \mid \mathscr{F}_{h}\right] \mid \mathscr{F}_{0}\right\} \\
& =E\left\{\int_{0}^{h} e^{-\rho t} \ln \left(c_{t}\right) d t\right. \\
& \left.+e^{-\rho h} E\left[\int_{h}^{+\infty} e^{-\rho(t-h)} \ln \left(c_{t}\right) d t \mid \mathscr{F}_{h}\right] \mid \mathscr{F}_{0}\right\} \\
& \leq E\left\{\int_{0}^{h} e^{-\rho t} \ln \left(c_{t}\right) d t+e^{-\rho h} V\left(s_{h}, q_{h}\right) \mid \mathscr{F}_{0}\right\} .
\end{aligned}
$$

Taking maximum on both sides, we get

$$
\begin{aligned}
& V(s, q) \\
& \quad \leq \max _{c_{t}, k_{t} \geq 0} E\left[\int_{0}^{h} e^{-\rho t} \ln \left(c_{t}\right) d t+e^{-\rho h} V\left(s_{h}, q_{h}\right) \mid \mathscr{F}_{0}\right] .
\end{aligned}
$$

(ii) For any $\varepsilon>0$, we can find $c_{t}^{\varepsilon}, k_{t}^{\varepsilon}$ such that

$$
V(s, q)-\varepsilon \leq E\left[\int_{0}^{h} e^{-\rho t} \ln \left(c_{t}^{\varepsilon}\right) d t \mid \mathscr{F}_{0}\right] .
$$

Denoting

$$
\begin{gathered}
\widehat{c}_{t}= \begin{cases}c_{t}, & 0<t \leq h \\
c_{t}^{\varepsilon}, & t>h,\end{cases} \\
\widehat{k}_{t}= \begin{cases}k_{t}, & 0<t \leq h \\
k_{t}^{\varepsilon}, & t>h,\end{cases}
\end{gathered}
$$

we have

$$
\begin{aligned}
V(s, q) \geq E\left[\int_{0}^{+\infty} e^{-\rho t} \ln \left(\widehat{c}_{t}\right) d t \mid \mathscr{F}_{0}\right] \\
=E\left\{E\left[\int_{0}^{+\infty} e^{-\rho t} \ln \left(\widehat{c}_{t}\right) d t \mid \mathscr{F}_{h}\right] \mid \mathscr{F}_{0}\right\} \\
=E\left\{E \left[\int_{0}^{h} e^{-\rho t} \ln \left(c_{t}\right) d t\right.\right. \\
\left.\left.+e^{-\rho h} \int_{h}^{+\infty} e^{-\rho(t-h)} \ln \left(c_{t}^{\varepsilon}\right) d t \mid \mathscr{F}_{h}\right] \mid \mathscr{F}_{0}\right\} \\
=E\left\{\int_{0}^{h} e^{-\rho t} \ln \left(c_{t}\right) d t\right. \\
\left.+e^{-\rho h} E\left[\int_{h}^{+\infty} e^{-\rho(t-h)} \ln \left(c_{t}^{\varepsilon}\right) d t \mid \mathscr{F}_{h}\right] \mid \mathscr{F}_{0}\right\} \\
\geq E\left[\int_{0}^{h} e^{-\rho t} \ln \left(c_{t}\right) d t+e^{-\rho h}\left(V\left(s_{h}, q_{h}\right)-\varepsilon\right) \mid \mathscr{F}_{0}\right] \\
=E\left[\int_{0}^{h} e^{-\rho t} \ln \left(c_{t}\right) d t+e^{-\rho h}\left(V\left(s_{h}, q_{h}\right)\right) \mid \mathscr{F}_{0}\right] \\
-\varepsilon .
\end{aligned}
$$

Letting $\varepsilon \longrightarrow 0$ and taking maximum, we obtain

$$
\begin{aligned}
& V(s, q) \\
& \geq \max _{c_{t}, k_{t}} E\left[\int_{0}^{h} e^{-\rho t} \ln \left(c_{t}\right) d t+e^{-\rho h}\left(V\left(s_{h}, q_{h}\right)\right) \mid \mathscr{F}_{0}\right] .
\end{aligned}
$$

Therefore, we get

$$
\begin{gathered}
V(s, q)=\max _{c_{t}, k_{t}} E\left[\int_{0}^{h} e^{-\rho t} \ln \left(c_{t}\right) d t\right. \\
\left.+e^{-\rho h}\left(V\left(s_{h}, q_{h}\right)\right) \mid \mathscr{F}_{0}\right] .
\end{gathered}
$$


Theorem 2. Assume $V(s, q) \in \mathscr{C}^{1,2}$, and then the value function $V(s, q)$ of a representative farmer satisfies the following Hamilton-Jacobi-Bellman (HJB) equation

$$
\begin{aligned}
\rho V & =\max _{c, k \geq 0}\left(\ln c+[k(a-q)-c] V_{s}+q \mu(q) V_{q}\right. \\
& \left.+\frac{1}{2} \sigma_{0}^{2} k^{2} V_{s s}+\frac{1}{2} q^{2 \beta} \sigma(q)^{2} V_{q q}+\sigma_{0} k q^{\beta} \sigma(q) V_{s q}\right) .
\end{aligned}
$$

Proof. Applying Itô's formula, we have

$$
\begin{aligned}
& d\left(e^{-\rho t} V\left(s_{t}, q_{t}\right)\right)=e^{-\rho t} \cdot(-\rho) \cdot V\left(s_{t}, q_{t}\right) d t \\
& +e^{-\rho t} d V\left(s_{t}, q_{t}\right)=e^{-\rho t}\left(-\rho V d t+\frac{\partial V}{\partial s} d s_{t}\right. \\
& +\frac{1}{2} \frac{\partial^{2} V}{\partial s^{2}} d\langle s\rangle_{t}+\frac{\partial V}{\partial q} d q_{t}+\frac{1}{2} \frac{\partial^{2} V}{\partial q^{2}} d\langle q\rangle_{t} \\
& \left.+\frac{\partial^{2} V}{\partial s \partial q} d\langle s, q\rangle_{t}\right)=e^{-\rho t} \cdot(-\rho V d t \\
& +V_{s}\left(k_{t}\left(a d t+\sigma_{0} d B_{t}-q_{t} d t\right)-c_{t} d t\right) \\
& +\frac{1}{2} V_{s s} k_{t}^{2} \sigma_{0}^{2} d t+V_{q}\left(\mu\left(q_{t}\right) q_{t} d t+\sigma\left(q_{t}\right) q_{t}^{\beta} d B_{t}\right) \\
& \left.+\frac{1}{2} V_{q q} \sigma^{2}\left(q_{t}\right) q_{t}^{2 \beta} d t+V_{s q} k_{t} \sigma_{0} q_{t}^{\beta} d t\right)=e^{-\rho t}(A d t \\
& \left.+B d B_{t}\right),
\end{aligned}
$$

where $B=\sigma_{0} k_{t} V_{s}+q_{t}^{\beta} \sigma\left(q_{t}\right) V_{q}$ and

$$
\begin{gathered}
A=-\rho V+\left[k_{t}\left(a-q_{t}\right)-c_{t}\right] V_{s} \\
+q_{t} \mu\left(q_{t}\right) V_{q}+\frac{1}{2} k_{t}^{2} \sigma_{0}^{2} V_{s s} \\
+\frac{1}{2} q_{t}^{2 \beta} \sigma^{2}\left(q_{t}\right) V_{q q} \\
+\sigma_{0} k_{t} q_{t}^{\beta} \sigma\left(q_{t}\right) V_{s q} \\
e^{-\rho h} V\left(s_{h}, q_{h}\right)=V(s, q)+\int_{0}^{h} d\left(e^{-\rho t} V\left(s_{t}, q_{t}\right)\right) \\
=V(s, q)+\int_{0}^{h} e^{-\rho t}\left(A d t+B d B_{t}\right) .
\end{gathered}
$$

Substituting (17) into (6), we obtain

$$
\begin{gathered}
V(s, q)=\max _{c_{t}, k_{t} \geq 0} E\left[\int_{0}^{h} e^{-\rho t} \ln \left(c_{t}\right) d t+V(s, q)\right. \\
\left.+\int_{0}^{h} e^{-\rho t}\left(A d t+B d B_{t}\right) \mid \mathscr{F}_{0}\right] .
\end{gathered}
$$

Namely,

$$
0=\max _{c_{t}, k_{t} \geq 0} E\left[\int_{0}^{h} e^{-\rho t}\left(\ln \left(c_{t}\right)+A\right) d t \mid \mathscr{F}_{0}\right] .
$$

Noticing that $\left(c_{t}, k_{t}\right)$ is procession on $[0, h]$, as $h \longrightarrow 0$, $c_{t}=c$, and $k_{t}=k$ (number, not process), dividing by $h>0$, setting $h \longrightarrow 0$, and using the mean value theorem, we have

$$
\begin{aligned}
\rho V & =\max _{c, k \geq 0}\left(\ln c+[k(a-q)-c] V_{s}+q \mu(q) V_{q}\right. \\
& \left.+\frac{1}{2} \sigma_{0}^{2} k^{2} V_{s s}+\frac{1}{2} q^{2 \beta} \sigma^{2}(q) V_{q q}+\sigma_{0} k q^{\beta} \sigma(q) V_{s q}\right),
\end{aligned}
$$

which completes the proof.

\section{Solution of the Equilibrium}

Note that the utility function is logarithmic and the HJB solution $V(s, q)$ is additively separable. The optimal consumption and scale of productivity are linear functions of the initial savings. We know that the feasible set of each farmer's decision $\left(c_{t}, k_{t}\right)$ is homothetic in his initial savings. Hence, we assume that the solution takes the form

$$
V(s, q)=\lambda \ln s+\varphi(q)
$$

where $\lambda$ is a constant and $\varphi(q)$ is a twice differentiable function of $q$. If $V(s, q)$ takes the form above, the property $V_{s q}=0$ can be found.

Theorem 3. If $V(s, q)=\lambda \ln s+\phi(q)$, then the optimal consumption and scale of productivity of each farmer are

$$
c^{*}(s, q)=\rho s,
$$

$$
k^{*}(s, q)=\frac{a-q}{\sigma_{0}^{2}} \cdot\left(\frac{V_{s s}}{V_{s}}\right)^{-1}=\frac{a-q}{\sigma_{0}^{2}} s .
$$

Proof. As the term $V_{s q}$ is vanished, the HJB equation becomes

$$
\begin{aligned}
\rho V & =\max _{c, k \geq 0}\left(\ln c+[k(a-q)-c] V_{s}+q \mu(q) V_{q}\right. \\
& \left.+\frac{1}{2} \sigma_{0}^{2} k^{2} V_{s s}+\frac{1}{2} q^{2 \beta} \sigma^{2}(q) V_{q q}\right) .
\end{aligned}
$$

Our aim is to maximize the function on the right side. To this end, we use the first-order condition with respect to $c$ and $k$, and we get

$$
\begin{aligned}
\frac{1}{c^{*}}-V_{s} & =0, \\
(a-q) V_{s}+k^{*} \sigma_{0}^{2} V_{s s} & =0 ;
\end{aligned}
$$

i.e.,

$$
\begin{aligned}
c^{*} & =\frac{1}{V_{s}}=\frac{s}{\lambda}, \\
k^{*} & =\frac{a-q}{\sigma_{0}^{2}} \cdot\left(-\frac{V_{s}}{V_{s s}}\right)=\frac{a-q}{\sigma_{0}^{2}} s .
\end{aligned}
$$


Putting the equations above into (14), we have

$$
\begin{aligned}
\rho(\lambda \ln s+\varphi(q))= & \ln \frac{s}{\lambda}+\left[\frac{(a-q)^{2}}{\sigma_{0}^{2}} s-\frac{s}{\lambda}\right] \cdot \frac{\lambda}{s} \\
& +q \mu(q) \varphi^{\prime}(q)+\frac{1}{2} \sigma_{0}^{2} \frac{(a-q)^{2}}{\sigma_{0}^{4}} s^{2} \\
& \cdot\left(-\frac{\lambda}{s^{2}}\right)+\frac{1}{2} q^{2 \beta} \sigma^{2}(q) \varphi^{\prime \prime}(q)
\end{aligned}
$$

Finally, we get

$$
\begin{aligned}
& (\rho \lambda-1) \ln s+\rho \varphi(q)+q \mu(q) \varphi^{\prime}(q) \\
& \quad-\frac{1}{2} q^{2 \beta} \sigma^{2}(q) \varphi^{\prime \prime}(q)-\frac{1}{2} \frac{(a-q)^{2} \lambda}{\sigma_{0}^{2}}+\ln \lambda+1 \\
& \quad=0 .
\end{aligned}
$$

The equation above holds for any $s$ and $q$. Then we must have

$$
\begin{aligned}
\rho \lambda-1 & =0, \\
\lambda & =\frac{1}{\rho} .
\end{aligned}
$$

The proof is completed.

As is well known, a representative farmer's optimal consumption is a linear function of his saving multiplied by a discount constant $\rho$. What is more important is that the farmer's optimal scale of productivity is also a linear function with respect to $s$, which is his induced risk tolerance $\left(-V_{s s} / V_{s}\right)^{-1}$. So the more saving a farmer has, the more land he will rent. In other words, if a farmer is out of money, he will rent less land from the landlord because he has more risk averseness.

Since the optimal consumption and the scale of rental land are determined, we can go on with the entire economy status. The aggregate stock of saving $S_{t}$ satisfies

$$
d S_{t}=K_{D}\left(q_{t}\right)\left(d y_{t}-q_{t} d t\right)-\rho S_{t} d t
$$

where $K_{D}\left(q_{t}\right)$ is the demands of lands for all farmers and follows

$$
K_{D}\left(q_{t}\right)=\frac{\left(a-q_{t}\right)}{\sigma_{0}^{2}} S_{t}
$$

To proceed, we give the definition of an equilibrium according to market clearing condition.

Definition 4. For any initial natural endowments and rental rate $q_{0}$, an equilibrium is defined by stochastic process with filtration generated by the Brownian motion $\left\{B_{t}, t \geq 0\right\}$ : the rental rate process $q_{t}$ and the aggregate saving process $S_{t}$ such that

(i) the land market clears: $K_{D}\left(q_{t}\right)=K_{S}\left(q_{t}\right)$,

(ii) the market for consumption goods clears.
If the equilibrium that is Markovian in the state $q_{t}$ exists, the aggregate stock of savings follows $S_{t}=S\left(q_{t}\right)$. Applying Itô's formula, we have

$$
\begin{aligned}
d S_{t}= & {\left[S^{\prime}\left(q_{t}\right) q_{t} \mu\left(q_{t}\right)+\frac{1}{2} q_{t}^{2} \sigma^{2}\left(q_{t}\right) S^{\prime \prime}\left(q_{t}\right)\right] d t } \\
& +\frac{a-q_{t}}{\sigma_{0}} S_{t} d B_{t} .
\end{aligned}
$$

Combining (32) with (30) yields

$$
\begin{aligned}
& \frac{(a-q)^{2}}{\sigma_{0}^{2}} S(q)-\rho S(q) \\
& \quad=q \mu(q) S^{\prime}(q)+\frac{1}{2} q^{2 \beta} \sigma^{2}(q) S^{\prime \prime}(q), \\
& \frac{a-q}{\sigma_{0}} S=\sigma(q) q S^{\prime}(q) .
\end{aligned}
$$

In addition, the clearing condition implies

$$
K_{S}(q)=\frac{(a-q)}{\sigma_{0}^{2}} S(q) .
$$

Theorem 5. There exists a unique Markov equilibrium in which the rental rate satisfies the following stochastic process:

$$
\frac{d q_{t}}{q_{t}}=\mu\left(q_{t}\right) d t+\sigma\left(q_{t}\right) q_{t}^{\beta-1} d B_{t}
$$

where

$$
\begin{aligned}
& \sigma(q)=\frac{(a-q)^{2}}{q^{\beta-1} \sigma_{0}\left[(a-q) \epsilon_{1}+q\right]}, \\
& \mu(q)=\sigma(q) \\
& \cdot q^{\beta-1}\left\{\frac{(a-q)^{2} \epsilon_{1}\left[(a-q)\left(2 \epsilon_{1}-\epsilon_{2}\right)+2 q\right]}{2 \sigma_{0}\left[(a-q) \epsilon_{1}+q\right]^{2}}\right. \\
&\left.-\frac{\rho \sigma_{0}}{a-q}\right\} .
\end{aligned}
$$

Proof. For convenience, we denote $\epsilon_{1}:=q\left(K_{S}^{\prime}(q) / K_{S}(q)\right)$ (the elasticity of land supply), $\epsilon_{2}:=q\left(K_{S}^{\prime \prime}(q) / K_{S}^{\prime}(q)\right)$. Equations (34) and (36) are obtained by solving (33) and (34) directly.

We now focus on the properties of (36) and (37). Comparing to the work of Klimenko et al. [10], the only difference is the $q^{\beta-1}$ part that occurred in the denominator of $\sigma(q)$. The paradox of the volatility in Brunnermeier and Sannikov [6] shows up as well. In (37), $\sigma_{0}$ has an inverse relationship with $\sigma(q)$ so that the high exogenous volatility coexists with the low endogenous volatility at the same time. Moreover, we can see that the endogenous volatility $\sigma$ is a linear function of $q^{2-\beta}$; i.e., $\sigma(q) \propto q^{2-\beta}$. For comparison, $\sigma(q)$ is a linear function of $q$; i.e., $\sigma(q) \propto q$ in [10]. We assume that $\beta$ takes 
value between 1 and 2 to ensure that $2-\beta$ is in the interval $(0,1)$. From the graph of function $f(x)=x^{(2-\beta)}$, we have that the curve of the endogenous volatility is antrorsely warped by the rental rate $q$. When $q$ is low, it has more impacts on $\sigma$. While $q$ is high, the influence will be pulled down. In Klimenko et al. [10], the influence is linear all the time.

\section{Properties of the Dynamics}

In this section, we are going to discuss the economy in the long term. All properties are determined by the dynamics of the rental rate $q_{t}$. Our main goal is to give deep insight into the process described in Theorem 2 . There are only two scenarios of $q_{t}$ that may occur: either the rental rate drops very close to 0 and remains at the state of 0 permanently or it fluctuates in the range of $(0, a)$. For the first case, we are supposed to investigate the attainability of the boundaries of the process $q_{t}$. For the latter, we will use the ergodic density function to discuss its main properties.

5.1. Ergodic Density Functions. If the ergodic density function of process $q_{t}$ does exist, we can derive it by solving a Kolmogorov's forward equation. Set

$$
\Lambda(q)=\frac{2 \mu(q)}{\sigma^{2}(q)} .
$$

As will be clarified, the existence of the ergodic density function and its properties depend on the parameter

$$
\Lambda(0)=\frac{2 \mu(0)}{\sigma^{2}(0)}=2\left(1-\frac{\rho}{\theta^{2}}\right) \epsilon_{1}(0)-\epsilon_{2}(0),
$$

where $\theta=a / \sigma_{0}$ signifies the risk-adjusted productivity of farmers.

Theorem 6. The properties of the economy depends on $\Lambda(0)$ :

(i) If $\Lambda(0) \leq 1$, the ergodic density function does not exist and the economy collapses asymptotically.

(ii) If $\Lambda(0)>1$, the process $q_{t}$ has the ergodic density distribution

$$
p(q)=\frac{C}{q^{2} \sigma^{2}(q)} e^{\int_{0}^{q}\left(2 \mu(s) / s \sigma^{2}(s)\right) d s},
$$

where $C$ is a constant such that $\int_{0}^{a} p(s) d s=1$. The boundary behaviour of the ergodic density function is characterized by the following:

(i) When $\Lambda(0) \geq 2, \lim _{q \rightarrow 0} p(q)$ is finite and is infinite otherwise.

(ii) $\lim _{q \rightarrow a} p(q)=0$.

Proof. If the process $q_{t}$ has a density $p(t, x)$, it satisfies the Kolmogorov differential equation

$$
\begin{aligned}
\frac{\partial p}{\partial t}(t, q)= & -\frac{\partial}{\partial q}[q \mu(q) p(t, q)] \\
& +\frac{1}{2} \frac{\partial^{2}}{\partial q^{2}}\left[q^{2} \sigma^{2}(q) p(t, q)\right] .
\end{aligned}
$$

If the Markov process $q_{t}$ is stationary, the stationary density $\psi(y)$ must satisfy

$$
\psi(y)=\int \psi(x) p(t, x) d x, \quad \forall t>0 .
$$

Mimicking the derivation of (41), we deduce $\psi(y)$ to satisfy

$$
0=-\frac{d}{d q}[q \mu(q) \psi(q)]+\frac{1}{2} \frac{d^{2}}{d q^{2}}\left[q^{2} \sigma^{2}(q) \psi(q)\right] .
$$

By calculation, we have

$$
\begin{aligned}
& \frac{d}{d q}[q \mu(y) \psi(q)]=\frac{1}{2} \frac{d^{2}}{d q^{2}}\left[q^{2} \sigma^{2}(q) \psi(q)\right], \\
& C_{1}+q \mu(q) \psi(q)=\frac{1}{2} \frac{d}{d q}\left[q^{2} \sigma^{2}(q) \psi(q)\right] .
\end{aligned}
$$

Denoting $\varsigma(\eta)=e^{-\int\left(2 \mu(\xi) / \xi \sigma^{2}(\xi)\right) d \xi}$ to be the integrating factor and $\zeta(q)=\int_{0}^{q} \varsigma(\eta) d \eta$, we have

$$
\begin{aligned}
& 2 C_{1} \varsigma(q)+2 \varsigma(q) q \mu(q) \psi(q) \\
& \quad \varsigma(q) \frac{d}{d q}\left[q^{2} \sigma^{2}(q) \psi(q)\right], \\
& \varsigma(q) \frac{d}{d q}\left[q^{2} \sigma^{2}(q) \psi(q)\right]-2 \varsigma(q) q \mu(q) \psi(q) \\
& \quad=C_{2} \varsigma(q), \quad\left(C_{2}=2 C_{1}\right) \\
& d\left[\varsigma(q) q^{2} \sigma^{2}(q) \psi(q)\right]=C_{2} \varsigma(q), \\
& \varsigma(q) q^{2} \sigma^{2}(q) \psi(q)=C_{2} \zeta(q)+C_{3}, \\
& \psi(q)=\frac{1}{\varsigma(q) q^{2} \sigma^{2}(q)}\left[C_{2} \zeta(q)+C_{3}\right] .
\end{aligned}
$$

Setting $m(q):=1 / \varsigma(q) q^{2} \sigma^{2}(q)$, we get

$$
\psi(q)=m(q)\left[C_{2} \zeta(q)+C_{3}\right],
$$

where $C_{2}$ and $C_{3}$ are constants to ensure that $\int_{0}^{a} \psi(q) d q=1$. $C_{2}$ is clarified to be 0 and for $q=a$ is unattainable in finite time. Therefore, the stationary density of the process $q_{t}$ is

$$
p(q)=\frac{C}{q^{2} \sigma^{2}(q)} e^{\int_{0}^{q}\left(2 \mu(s) / s \sigma^{2}(s)\right) d s},
$$

where $C$ is a constant to guarantee that $\int_{0}^{a} p(q) d q=1$.

Now we investigate the properties of $p(q)$ at both ends of the support $[0, a]$. In the right neighborhood of the state 0 : $\Theta_{0}=(0, \varepsilon]$, the drift and volatility part of the process $q_{t}$ are approximated by

$$
\begin{aligned}
& \sigma(0) \approx \frac{a}{\sigma_{0} \epsilon_{1}(0)}, \\
& \mu(0) \approx \sigma(0)\left\{\frac{a^{2}\left[2 \epsilon_{1}(0)-\epsilon_{2}(0)\right]-2 \rho \sigma_{0}^{2} \epsilon_{1}(0)}{2 a \sigma_{0} \epsilon_{1}(0)}\right\} .
\end{aligned}
$$


For $0<q_{0}<q<\varepsilon$, the ergodic density function of $q_{t}$ is approximated by

$$
\begin{aligned}
p(q) & \approx \frac{C}{q^{2} \sigma^{2}(0)} e^{\int_{q_{0}}^{q}(2 \mu(0) / s \sigma(0)) d s} \\
& =\frac{C}{q^{2} \sigma^{2}(0)} e^{\int_{q_{0}}^{q}(\Lambda(0) / s) d s}=N q^{\Lambda(0)-2}
\end{aligned}
$$

where $N=C / q_{0}^{\Lambda(0)} \sigma^{2}(0)$ is a constant.

It is seen that $\lim _{q \rightarrow 0} p(q)<\infty$ if and only if $\Lambda(0) \geq 2$. Moreover, for any $0<q_{0}<q<\varepsilon$, in case that the probability distribution does not degenerate when $q \longrightarrow 0$, we have

$$
\int_{q_{0}}^{q} p(s) d s \approx \frac{N}{\Lambda(0)-1} q^{\Lambda(0)-1}<\infty .
$$

This condition holds if and only if $\Lambda(0)>1$. If $\Lambda(0)<1$, the ergodic distribution degenerates and the process $q_{t}$ ends up in a very close neighborhood of 0 with probability close to 1.

We now talk about the upper boundary behaviour of process $q_{t}$. Consider the left neighborhood of the state $a$ : $\Theta_{0}=[a-\varepsilon, a)$. In this status, the drift and volatility of process $q_{t}$ are approximated by (see details in Section 5.2)

$$
\begin{aligned}
q \mu(q) & =-\rho(a-q), \\
q^{\beta} \sigma(q) & =\frac{(a-q)^{2}}{\sigma_{0}} .
\end{aligned}
$$

The ergodic density function of $q_{t}$ is approximated by

$$
\begin{array}{r}
p(q) \propto \frac{1}{(a-q)^{4}} e^{-1 /(a-q)^{2}}, \\
\lim _{q \rightarrow a} \frac{1}{(a-q)^{4}} e^{-1 /(a-q)^{2}} \stackrel{x=1 /(a-q)^{2}}{=} \lim _{x \longrightarrow \infty} \frac{x^{2}}{e^{x}}=0 .
\end{array}
$$

This establishes that the density of $q_{t}$ vanishes on the occasion that the process $q_{t}$ goes very close to its upper boundary $a$.

5.2. Attainability of the Boundaries. For two natural boundaries: $q=0$ and $\bar{q}=a$, we consider the rental rate to stay in the neighborhood of 0 . If the economy could reach this state, it means that all farmers have no savings and they choose to rent no land. The landlords will cultivate all the land by themselves. Next, we consider the situation when $q_{t}$ fluctuates in the neighborhood of $a$. In this case, all landlords are happy to rent their land to farmers. So, the aggregate supply of land in the economy is 1 . In the view of the land market clearing condition, the demand of land is also 1. Equation (32) implies that the aggregate saving of farmers is approximated by $1 /(a-$ $q)$. Inserting this approximation into (32), the dynamics of $q_{t}$ in the neighborhood of $a$ are driven by

$$
d q_{t}=-\left(a-q_{t}\right)\left(\rho d t-\frac{a-q_{t}}{\sigma_{0}} d B_{t}\right)
$$

For further verification, we denote some functions according to boundary classification rules in Chapter 15 in [11] the following:

(i) Scale Function: $\zeta[b, q]=\int_{b}^{q} \varsigma(\eta) d \eta ; \varsigma(\eta)=e^{-\int^{\eta}(2 \mu(\xi) /}$ $\left.\xi \sigma^{2}(\xi)\right) d \xi$.

(ii) Speed Function: $M[b, q]=\int_{b}^{q} m(\eta) d \eta ; m(\eta)=1 /$ $\eta^{2} \varsigma(\eta) \sigma^{2}(\eta)$

(iii) $\sum(\iota)=\int_{\iota}^{q} M[\eta, q] d \zeta(\eta)$.

Definition 7 (see [11]). A boundary $l$ is attracting if $\lim _{q \rightarrow l} \varsigma[l, q]<\infty$.

Definition 8 (see [11]). A boundary $l$ is said to be

(i) attainable if $\sum(l)<\infty$,

(ii) unattainable if $\sum(l)=\infty$.

Lemma 9 (see [11]). A nonattracting boundary is unattainable in finite time; i.e., $\lim _{b \rightarrow l} \varsigma[b, q]=\infty$ implies $\lim _{b \rightarrow l} \sum(b)=$ $\infty$.

Theorem 10. The states $l=0$ and $r=a$ are both unattainable by process $q_{t}$ in finite time.

Proof. (i) For $0<q_{0}<q<\varepsilon$, we have

$$
\begin{aligned}
& \varsigma(\eta)=e^{-(\Lambda(0) / \xi) d \xi}=\left(\frac{q_{0}}{\eta}\right)^{\Lambda(0)}, \\
& m(\eta)=\frac{\eta^{\Lambda(0)}}{q_{0}^{\Lambda(0)} \eta^{2} \sigma^{2}(0)}=\frac{1}{q_{0}^{\Lambda(0)} \sigma^{2}(0)} \eta^{\Lambda(0)-2} \\
& =\widehat{A}_{0} \eta^{\Lambda(0)-2} \text {, } \\
& \widehat{A}_{0}=\frac{1}{q_{0}^{\Lambda(0)} \sigma^{2}(0)}, \\
& M[b, q]=\int_{b}^{q} m(\eta) d \eta=\int_{b}^{q} \widehat{A}_{0} \eta^{\Lambda(0)-2} d \eta \\
& =\frac{\widehat{A}_{0}}{\Lambda(0)-1} \cdot\left(q^{\Lambda(0)-1}-b^{\Lambda(0)-1}\right) \text {, } \\
& \sum(l)=\int_{l}^{q} M[b, q] d \zeta(b) \\
& =\int_{l}^{q} \frac{\widehat{A}_{0}}{\Lambda(0)-1} \cdot\left(q^{\Lambda(0)-1}-b^{\Lambda(0)-1}\right) \cdot\left(\frac{q_{0}}{b}\right)^{\Lambda(0)} d b \\
& =\frac{1}{[\Lambda(0)-1]^{2} \sigma^{2}(0)} \\
& \cdot\left[(\Lambda(0)-1) \ln \frac{l}{q}+\left(\frac{q}{l}\right)^{\Lambda(0)-1}-1\right] .
\end{aligned}
$$

If $\Lambda(0)>1$, we get

$$
\begin{gathered}
\lim _{l \rightarrow 0}\left(\frac{q}{l}\right)^{\Lambda(0)-1}=\infty \Longrightarrow \\
\lim _{l \rightarrow 0} \sum(l)=\infty .
\end{gathered}
$$


If $\Lambda(0) \leq 1$, we obtain

$$
\begin{aligned}
\lim _{l \rightarrow 0}(\Lambda(0)-1) \ln \frac{l}{q} & =\infty \Longrightarrow \\
\lim _{l \longrightarrow 0} \sum(l) & =\infty,
\end{aligned}
$$

which implies that the lower boundary $l=0$ is unattainable in finite time.

(ii) For $a-\varepsilon<q<q_{0}<a$, we have $q \mu(q)=-\rho(a-q)$, $q \sigma(q)=(a-q)^{2} / \sigma_{0}$, and

$$
\begin{aligned}
\varsigma(q) & =e^{-\int_{q_{0}}^{q}\left(2 \mu(\xi) / \xi \sigma^{2}(\xi)\right) d \xi}=e^{\int_{q_{0}}^{q}\left(2 \rho \sigma_{0}^{2} /(a-\xi)^{3}\right) d \xi} \\
& =\widetilde{A}_{0} e^{\rho \sigma_{0}^{2} /(a-q)^{2}},
\end{aligned}
$$

where $\widetilde{A_{0}}=\left[\rho \sigma_{0}^{2} /\left(a-q_{0}\right)^{2}\right]^{-1}$.

Thus, we have

$$
\zeta[q, r]=\int_{q}^{r} \varsigma(\eta) d \eta=\widetilde{A}_{0} \int_{q}^{r} e^{\rho \sigma_{0}^{2} /(a-\eta)^{2}} d \eta .
$$

Changing the variable by denoting $x=\sqrt{\rho} \sigma_{0} /(a-\eta), x_{q}=$ $\sqrt{\rho} \sigma_{0} /(a-q)$, and $x_{r}=\sqrt{\rho} \sigma_{0} /(a-r)$, we get

$$
\begin{aligned}
d \eta & =\frac{\sqrt{\rho} \sigma_{0}}{x^{2}} d x, \\
\zeta[q, r] & =\widetilde{A}_{0} \int_{x_{q}}^{x_{r}} \sqrt{\rho} \sigma_{0} \frac{e^{x^{2}}}{x^{2}} d x \\
& =\widetilde{A}_{0} \sqrt{\rho} \sigma_{0}\left[-\left.\frac{e^{x^{2}}}{x}\right|_{x_{q}} ^{x_{r}}+\int_{x_{q}}^{x_{r}} 2 e^{x^{2}} d x\right] \\
& =\left.\widetilde{A}_{0} \sqrt{\rho} \sigma_{0}\left(-\frac{e^{x^{2}}}{x}+\sqrt{\pi} E r f i(x)\right)\right|_{x_{q}} ^{x_{r}},
\end{aligned}
$$

where $\operatorname{Erfi}(x)$ is the imaginary error function which has derivative $d \operatorname{Erfix} / d x=(2 / \sqrt{\pi}) e^{x^{2}}$.

It can be known that $\zeta[q, r]$ goes to $\infty$ as $r$ tends to $a$. From Lemma 1 and Definition 7 , the state $a$ is unattainable in finite time.

In this section, we mainly talk about the properties of the ergodic density function. We get similar results as that in Klimenko et al. [10]. For the sake of explanation, we are supposed to track down to the model of process of $q_{t}$. The rental rate $q$ has a nonlinear influence on the parameter $\sigma$, which is explicitly described by $\sigma(q) \propto q^{(2-\beta)}$. If we track down to the stochastic differential equation of the process $q_{t}$, we find that the influence is counteracted by the SDE itself. This is why we get the same results. In the rest of our paper, we will talk about the process of $q_{t}$ in detail.

\section{Long-Run Average Behaviour, Welfare Loss, and Poverty Trap}

6.1. Long-Run Average Behaviour. When $\Lambda(0)>1$, we know that there exists an ergodic density function of the equilibrium rental rate process $q_{t}$. As the time goes to infinity, the time average of the stochastic variable converges. Therefore, the long-run average of $q_{t}$ is computed by

$$
\widetilde{q}=\lim _{T \rightarrow \infty} \int_{0}^{T} p_{s} d s=\int_{0}^{a} q p(q) d q
$$

If $\Lambda(0) \leq 1$, the ergodic distribution degenerates and the process $q_{t}$ ends up in a very close neighborhood of 0 with probability near to 1 , which indicates $\widetilde{q}=0$.

6.2. Welfare Loss. When the economy is frictionless, all the land is cultivated by the farmers. While the economy has extreme friction, we know that only part of lands is rented by farmers. The welfare loss is clarified to be the difference between the aggregate output in two cases. We are going to give an explicit formula of welfare loss and discuss its main property.

If $\widetilde{\alpha}>q_{t}$, the landlord will cultivate himself. Otherwise, he rents the land to farmers. Once the rental rate $q_{t}$ is determined, the total output process is an integral with respect to the level of $\widetilde{\alpha}$

$$
d Y_{t}=d y_{t} \int_{0}^{q_{t}} d\left[K_{S}(\widetilde{\alpha})\right]+d \tilde{y}_{t} \int_{q_{t}}^{a} d\left[K_{S}(\widetilde{\alpha})\right]
$$

In a frictionless economy, the rental rate is fixed at $q_{t}=a$ and all land is cultivated by farmers. The possible maximum output is

$$
d Y_{t}^{P M}=d y_{t} \int_{0}^{a} d\left[K_{S}(\widetilde{\alpha})\right]=a d t+\sigma_{0} d B_{t}
$$

The possible maximum output minus the total output is the welfare loss in the economy

$$
l\left(q_{t}\right)=d Y^{P M}-d Y_{t}=\int_{q_{t}}^{a}(a-\widetilde{\alpha}) d\left[K_{S}(\widetilde{\alpha})\right] .
$$

Because the ergodic density of $q_{t}$ does not exist when $\Lambda(0) \leq$ 1 , we only consider the situation when $\Lambda>1$. In this case, we compute the time average loss by ergodic property

$$
\tilde{l}=\lim _{T \rightarrow \infty} \frac{1}{T} \int_{0}^{T} l_{s} d s=\int_{0}^{a} l(q) p(q) d q .
$$

6.3. Poverty Trap. Following the ideas in [10], we take the constant-elasticity specification of land supply: $K_{S}(q)=$ $(q / a)^{\gamma}$, where $\gamma>0$. We derive that $\epsilon_{1}(q)=q\left(K_{S}^{\prime}(q) / K_{S}(q)\right)=$ $\gamma$ and $\epsilon_{2}=q\left(K_{S}^{\prime \prime}(q) / K_{S}^{\prime}(q)\right)=\gamma-1$. Substituting the values of $\epsilon_{1}$ and $\epsilon_{2}$ into (36) and (37), we have

$$
\begin{aligned}
& \sigma(q)=\frac{(a-q)^{2}}{q^{\beta-1} \sigma_{0}[(a-q) \gamma+q]}, \\
& \mu(q)=\sigma(q) \\
& \quad \cdot q^{\beta-1}\left\{\frac{\gamma(a-q)^{2}[(a-q) \gamma+a+q]}{2 \sigma_{0}[(a-q) \gamma+q]^{2}}-\frac{\rho \sigma_{0}}{a-q}\right\}, \\
& \Lambda(0)=\left(1-\frac{2 \rho}{\theta^{2}}\right) \gamma+1 .
\end{aligned}
$$


The poverty trap is mainly caused by the inverse relationship between the endogenous and the exogenous volatility. This occurs in our result as well. When the exogenous volatility $\sigma_{0}$ is low, a small shock in the economy gives rise to the big change of endogenous volatility $\sigma$. As a result, the economy will be trapped in a less productive level permanently (see details in Section 4.2 in [10]).

The process of $q_{t}$ is

$$
\frac{d q_{t}}{q_{t}}=\frac{\gamma\left(a-q_{t}\right)^{4}\left[\left(a-q_{t}\right) \gamma+a+q_{t}\right]-2 \rho \sigma_{0}^{2}\left(a-q_{t}\right)\left[\gamma\left(a-q_{t}\right)+q_{t}\right]^{2}}{2 \sigma_{0}^{2}\left[\gamma\left(a-q_{t}\right)+q_{t}\right]^{3}} d t+\frac{\left(a-q_{t}\right)^{2}}{\sigma_{0}\left[\gamma\left(a-q_{t}\right)+q_{t}\right]} d B_{t} .
$$

In (68), the parameter $\beta$ no longer exists. We are inspired whether people determine behaviour of the market or are controlled by it. Even if we take the agents' psychological anticipation (with parameter $\beta$ ) into consideration in our model, the rental market always runs in its own way (without $\beta)$. This is very meaningful.

\section{Conclusion}

In this paper, we investigate an economy model with extreme friction. In the economy, farmers have to choose their optimal scale of productivity to gain the maximum utility. We obtain conclusions similar to those in Klimenko et al. [10], such as poverty trap, existence conditions of the ergodic density function, and welfare loss. We take the farmers' psychological anticipations into account and adopt a different model of the rental rate process. The closed form of the endogenous volatility is obtained. We analyze how the rental rate influences the endogenous volatility. We find that this consideration does not show up in rental rate process ( $\beta$ does not appear in (68)). This gives us an inspiration that the capital market has some superior powers that are not up to the agents therein.

\section{Data Availability}

The data used to support the findings of this study are available from the corresponding author upon request.

\section{Conflicts of Interest}

The authors declare that there are no conflicts of interest regarding the publication of this paper.

\section{Authors' Contributions}

The article is a joint work of two authors who contributed equally to the final version of the paper. All authors read and approved the final manuscript.

\section{Acknowledgments}

This work is supported by the Fundamental Research Funds for the Central Universities (JBK120504).

\section{References}

[1] C. Kindleberger, A Financial History of Western Europe, vol. 53, Oxfod University Press, 2nd edition, 1993.
[2] B. Bernanke and M. Gertler, "Agency costs, net worth, and business fluctuations," American Economic Review, vol. 79, no. 1, pp. 14-31, 1989.

[3] N. Kiyotaki and J. Moore, "Credit cycles," Journal of Political Economy, vol. 105, no. 2, pp. 211-248, 1997.

[4] Z. He and A. Krishnamurthy, "A model of capital and crises," Review of Economic Studies, vol. 79, no. 2, pp. 735-777, 2012.

[5] Z. He and A. Krishnamurthy, "Intermediary asset prices," American Economic Review, vol. 103, no. 2, pp. 1-43, 2013.

[6] M. K. Brunnermeier and Y. Sannikov, "A macroeconomic model with a financial sector," American Economic Review, vol. 104, no. 2, pp. 379-421, 2014.

[7] X.-J. Xie and W.-Q. Li, "Output-feedback control of a class of high-order stochastic nonlinear systems," International Journal of Control, vol. 82, no. 9, pp. 1692-1705, 2009.

[8] X. Yu and X.-J. Xie, "Output feedback regulation of stochastic nonlinear systems with stochastic iISS inverse dynamics," Institute of Electrical and Electronics Engineers Transactions on Automatic Control, vol. 55, no. 2, pp. 304-320, 2010.

[9] X. Yu, X.-J. Xie, and Y.-Q. Wu, "Decentralized adaptive output-feedback control for stochastic interconnected systems with stochastic unmodeled dynamic interactions," International Journal of Adaptive Control and Signal Processing, vol. 25, no. 8, pp. 740-757, 2011.

[10] N. Klimenko, S. Pfeil, and J.-C. Rochet, "A simple macroeconomic model with extreme financial frictions," Journal of Mathematical Economics, vol. 68, pp. 92-102, 2017.

[11] S. Karlin and H. M. Taylor, A Second Course in Stochastic Processes, Academic Press, New York, NY, USA, 1981. 


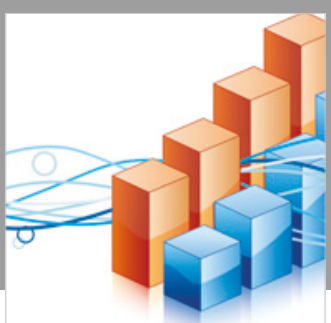

Advances in

Operations Research

\section{-n-m}
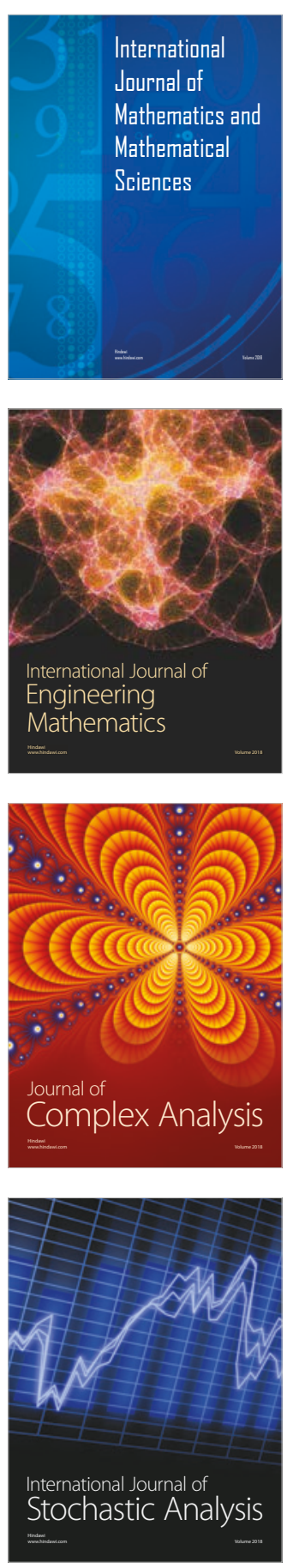
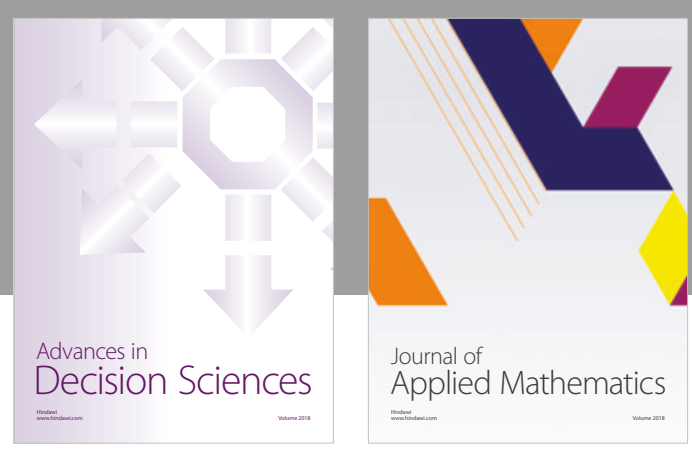

Journal of

Applied Mathematics
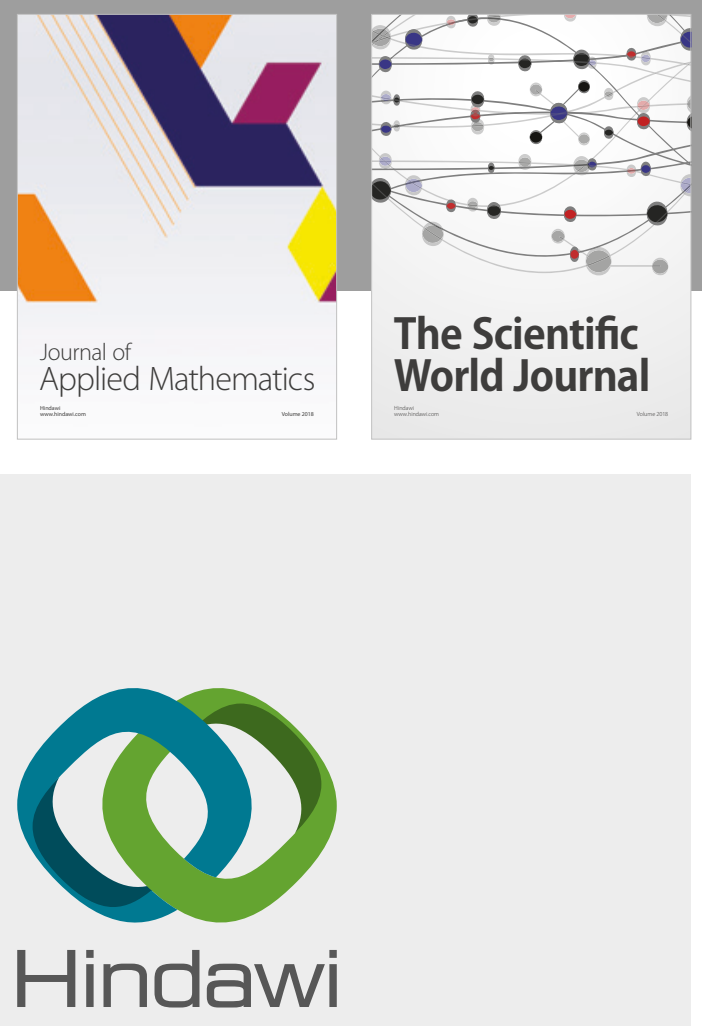

Submit your manuscripts at

www.hindawi.com

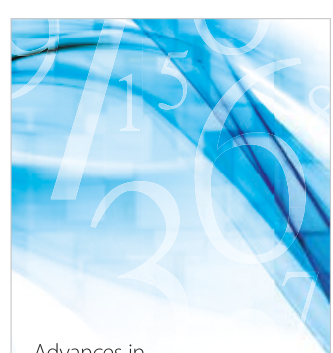

Advances in
Numerical Analysis
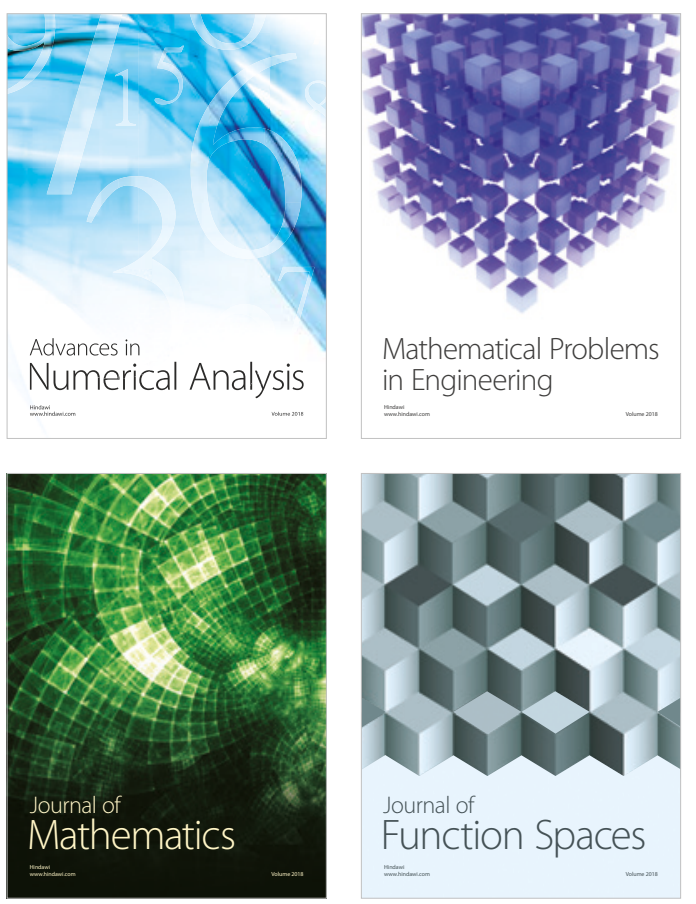

Mathematical Problems in Engineering

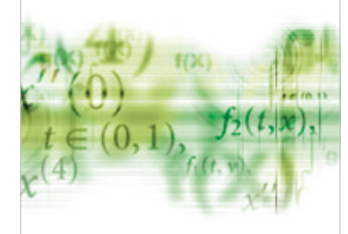

International Journal of

Differential Equations

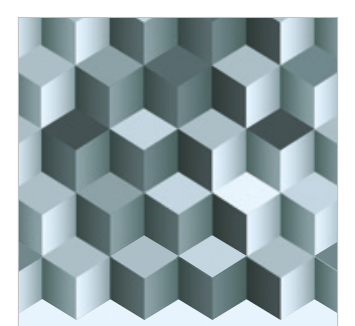

Journal of

Function Spaces

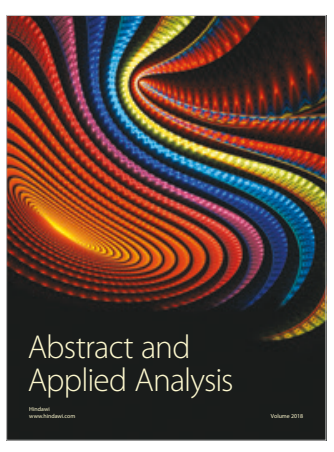

The Scientific

World Journal

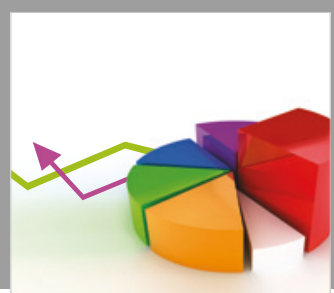

Journal of

Probability and Statistics
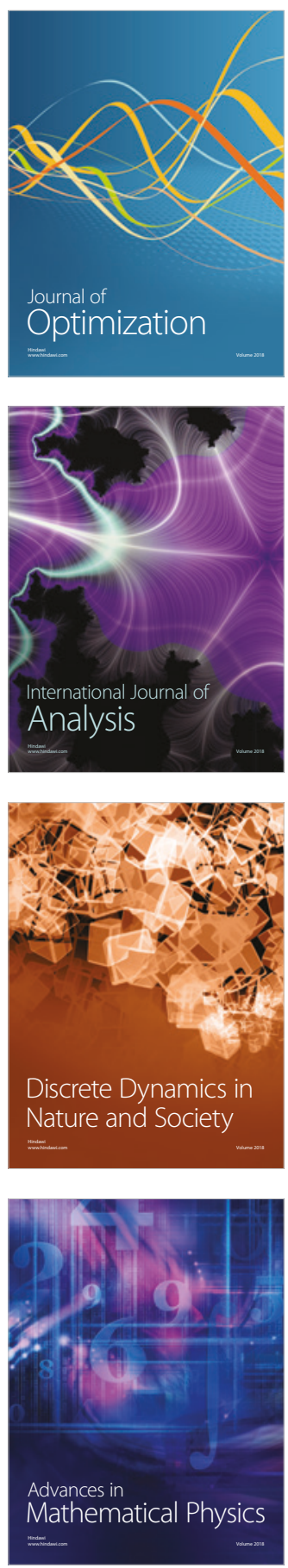